\title{
The Acute Effect of Exercise on Executive Function and Attention: Resistance Versus Aerobic Exercise
}

\author{
Sharon Tsuk', Yael Netz', Ayelet Dunsky', Aviva Zeev' ${ }^{1}$ Rafi Carasso², Tzvi Dwolatzky ${ }^{3}$, Riki \\ Salem', Shai Behar', and Arie Rotstein' \\ The Zinman College of Physical Education and Sport Sciences, Wingate Institute, Israel \\ ${ }^{2}$ The Hillel Yaffe Medical Center, Hadera, Ilsrael \\ ${ }^{3}$ Geriatric Unit, Rambam Health Care Campus; Ruth and Bruce Rappaport Faculty of Medicine, Technion-Israel Institute of \\ Technology, Haifa, israel
}

ABSTRACT

Acute aerobic exercise was shown to enhance such cognitive functions as executive function (EF) and attention. Acute resistance exercise was also shown to enhance cognitive functions, however, only few studies directly compared these two exercise modalities. The aim of this study was to evaluate the acute effect of a typical moderate intensity resistance exercise session as compared to a typical moderate intensity aerobic session, on executive function and attention. A counterbalanced repeated measures experimental design was applied. Forty physical education students ( 21 women; 19 men, age $=25.7 \pm 2.84$ years) were tested before and after three sessions: aerobic, resistance, and control. Each session consisted of 30 minutes of exercise or a rest. Executive function and attention were assessed by components of the computerized Stroop Catch game and Go-NoGo cognitive tests. A two-way ANOVA showed a greater increase in attention scores after the resistance sessions $(p<.05)$ compared to the control condition. Attention scores in the aerobic sessions showed a trend toward improvement but did not reach statistical significance. Scores of EF significantly increased, both after the resistance session and the aerobic session $(p<.05)$, but not after rest in the control condition. Our findings show that an acute session of resistance exercise increased both Attention and EF test scores, while an aerobic exercise session improved only the EF scores.

strength

\section{INTRODUCTION}

In a recent review, Basso and Suzuki (2017) summarized a large body of research describing the significant changes that occur at the cognitive, behavioral, neurophysiological, and neurochemical levels after a single bout of physical exercise. However, most of the interventions they describe are in the domain of aerobic exercise of various durations and intensities. For example, they provide ample evidence of specific effects of acute aerobic exercise on executive function (EF) and attention. Acute aerobic exercise is a one-session intervention, which may involve 25 min of treadmill or cycle exercise at a target heart rate of $60 \%$ of the heart rate reserve (Bullock \& Giesbrecht, 2014;
Dunsky et al., 2017; Netz et al., 2016; Tine, 2014; Zimmer et al., 2016). Importantly, acute aerobic exercise differs from acute resistance exercise in the immediate acute physiological responses, as well as in the long-term physiological adaptations (Knuttgen, 2007).

Acute moderate-intensity aerobic exercise was found to improve $\mathrm{EF}$, as indicated by higher scores in the interference phase of the Stroop test in endurance athletes (Hogervorst, Riedel, Jeukendrup, \& Jolles,

Corresponding author: Sharon Tsuk, The Zinman College of Physical Education and Sport Sciences, Wingate Institute, Israel 4290200. E-mail: sharonts@wincol.ac.il 
1996), and in young adults (Tam, 2013). Interestingly, the improvement in the interference phase of executive control in young athletes was explained by improved attention (Chang, Pesce, Chiang, Kuo, \& Fong, 2015). Not all studies showed positive effects of aerobic exercise on the interference test. For example, in older participants, a positive effect was reported only in the noninterference phase (Barella, Etnier, \& Chang, 2010). Relatively fewer studies examined the effects of acute resistance exercise. It was shown that in adults with impaired cognitive function, resistance exercise improved global cognitive function and EF (Fiatarone Singh et al., 2014). In middle-aged participants, Stroop test scores were improved after an acute moderate resistance exercise (Chang \& Etnier, 2009).

In a meta-analysis on the effects of acute exercise on cognitive functions, Chang, Ku, Tomporowski, Chen, and Huang (2012) name a number of variables that may moderate these effects, including: mode of exercise (e.g., aerobic or resistance exercise), duration and intensity of the exercise, time of testing (during or after the exercise bout), participants' age and baseline health, fitness and cognitive functioning, and the complexity and type of the tested task. Thus, it is not surprising that many inconsistencies may be found in the literature (Chang, Labban, Gapin, \& Etnier, 2012; Johnson et al., 2016).

Studies designed to directly compare the effects of acute resistance exercise with those of acute aerobic exercise on cognitive function of young, healthy subjects are scarce. Positive effects of acute resistance and aerobic exercise on EF were shown in older women (Alves et al., 2012) and high school students (Harveson et al., 2016). However, a study performed on young people aged $20.2 \pm 0.3$ years showed improvement in memory only following aerobic exercise, and not resistance exercise (Pontifex, Hillman, Fernhall, Thompson, \& Valentini, 2009).

Recently, Dunsky et al. (2017) examined the influence of acute aerobic versus resistance exercise session on attention and executive function in healthy middle-aged participants. Their main finding was that changes in attention scores following aerobic exercise were significantly higher than those following the control condition.

Johnson et al. (2016) compared the cognitive performance of older adults aged 71-72 years using the Stroop test before and after an acute aerobic or resistance exercise session. They also give a review of the findings concerning the effects of acute aerobic and strength exercise, and discuss the difficulties in comparing their effects on cognitive performance. They also point out the various factors which may influence the results of such investigations (Johnson et al., 2016).

The limited amount of data directly comparing the effects of acute resistance exercise and acute aerobic exercise on cognitive function of young and healthy participants has led to the objective of the current study. Our purpose was to compare the acute effects of a typical moderate-intensity resistance exercise session with the acute effects of a typical moderate-intensity aerobic exercise on cognitive functions relating to EF and attention in a group of young, healthy physical education students familiar with both training modes.

Exercise may prove to be an effective therapeutic tool for delaying or treating cognitive decline, and may also prove to be an effective tool for the enhancement of cognitive functions in healthy individuals. A single bout of acute exercise may also prove to be a practical tool for improving functioning in stressful situations or in an academic environment during learning and test-taking. Resistance or strength training is a popular training mode, and many young and healthy people prefer this training mode and limit their involvement in aerobic training.

\section{METHOD}

All participants provided written informed consent for participation in the study, and the study was approved by the Ethics Committee of the Hillel Yaffe Medical Center (Hadera, Israel).

\section{Participants}

Participants were volunteers recruited from students enrolled in a degree program in physical education at the Wingate Academic College. Forty physical education students (21 women), aged 25.7 \pm 2.84 years, participated in the study. All of the students were familiar with both training modes. Inclusion criteria were: non-smoking, no neurological or psychiatric disease, no prescribed medications that might alter cognitive function, and no head injury or long-term hospitalization in the previous three months.

\section{Procedure}

Participants visited the lab four times, each visit no more than one week from the previous visit. They were asked not to engage in any structured exercise on the day of their testing session, and not to consume caffeine for at least two hours prior to the session.

The visits were in the following order:

\section{SESSION 1: BASELINE TESTING}

Participants completed an informed consent form and then performed an aerobic fitness trial and resistance exercise trial for assessment. They then performed a familiarization trial of the cognitive tests.

\section{SESSION 2: AEROBIC FITNESS ASSESMENT}

A progressive, maximal cycle exercise test (modified from the American College of Sports Medicine, ACSM, in 2010; ACSM, Thompson, Gordon, \& Pescatello, 2010) on a Monark 834k cycle ergometer (Monark, Stockholm) was performed. The test consisted of a continuous incremental protocol leading to volitional exhaustion. After a 2 min warm-up performed by the participants at $50 \mathrm{~W}$, the exercise workload was increased by $25 \mathrm{~W}$ every 2 min until exhaustion. Participants were all verbally encouraged to achieve their maximal performance. The test was used to determine an individual workload for the aerobic exercise session (60\% of maximal workload).

\section{SESSION 3: RESISTANCE FITNESS TEST}

After $30 \mathrm{~min}$ of rest, the participants performed a resistance exercise test. The test was used to determine a maximum of 15 repetitions (15-RM), which represents $60 \%$ of 1-RM (Fleck \& Kraemer, 
2014). The test was performed for six exercises: chest press, leg press, vertical traction, chest pull, leg extension, and shoulder press, using a Body-Solid EXM3000S multi-station gym machine. The procedure of this test was standardized, and was conducted by the same person for all participants. A 5 min rest period was given between the various exercise modes.

\section{Cognitive Assessment}

Cognitive function was evaluated by a computerized cognitive evaluation program, which included three tests: the Stroop test, the Go-NoGo test, and the Catch Game test (Mindstreams, NeuroTrax Corp., NJ, USA).

Participants were administered the tests in the same fixed order. Testing time was approximately 15 minutes. There were no rest periods between the three tests.

\section{GO-NOGO TEST}

A timed continuous performance test. The test measures components of attention and executive function, and also evaluates response time and response inhibition. The participants were presented with a series of large colored squares on a computer screen at variable sequences. Each square was one of four colors. The participants were instructed to respond as quickly as possible by pressing a computer mouse button if the square appeared in any color but red. This test included 30 trials. The variables measured included: accuracy, response time and its associated variance, a composite score computed as accuracy divided by response time, number of errors of omission, number of errors of commission, and response time associated with errors of commission. Omission errors are assumed to reflect deficient sustained attention or vigilance; commission errors - a combination of underlying processes, including impulsivity and inattention/memory deficit (Halperin, Wolf, Greenblatt, \& Young, 1991).

\section{STROOP TEST}

A timed test of response inhibition and set shifting. The test measures cognitive functions related to attention and executive functions. This test was divided into three stages (levels):

Stage 1 (no interference stage): The participants were presented with a word in colored letters on a computer screen - the word did not name a color. Following a brief delay, the participants were presented with a pair of colored squares. They were instructed to choose as quickly as possible, by pressing a computer mouse button, the square that was the same color as the letters of the word presented. This stage included 10 trials.

Stage 2 (no interference stage): The participants were presented with a word on a computer screen that names a color in white letters. They were instructed to choose the color, by pressing a computer mouse button, from a list which represented the meaning of the word. This stage included 15 trials.

Stage 3: (with interference stage): Participants were presented with a word on a computer screen that names a color written with letters of a color other than that named by the word's meaning. They were instructed to choose, by pressing a computer mouse button, only the squares colored as presented by the letter's color. This stage included 15 trials.

Outcome parameters for each phase included accuracy, response time and its associated variance, and a composite score computed as accuracy divided by response time.

\section{CATCH GAME TEST}

This test measures motor planning involving hand-eye coordination and rapid responses. The test requires participants to catch a falling object on the computer screen by moving a paddle horizontally so that it can be positioned directly in the path of the falling object. This test included 20 trials. Outcome parameters included response time and associated variance for the first move, number of direction changes per trial, error for missed catches, and a total performance score.

These three cognitive tests are part of the Mindstreams (NeuroTrax Corp., NJ, USA) computerized battery that utilizes novel adaptations of traditional neuropsychological tests. Previous research has shown that these tests have good concurrent validity and reliability, and are highly correlated with performance on traditional neuropsychological batteries (Dwolatzky et al., 2003). This battery was already used with young, healthy adults (Doniger, Simon, \& Schweiger, 2008). The battery is simple to use, requires no previous computer experience, and is web-based with a central database. All responses are made using either the computer mouse or the number pad on the keyboard.

\section{Outcome Parameters}

The outcome parameters include four scores: accuracy (number of correct responses); response time (RT); SD of RT; and, given the speed-accuracy tradeoff, a performance index, computed as (accu$\mathrm{racy} / \mathrm{RT}) \times 100$, that is computed to assess performance in terms of both accuracy and response time.

The scores of the outcome parameters of the three tests are averaged to produce two summary scores, each indexing a different cognitive domain, as follows:

\section{ATTENTION}

Mean RTs for the Go-NoGo test and the no-interference (meaning) phase of the Stroop test, and mean $S D$ of RT for the Go-NoGo test. These variables are related to attention, as rapid responses are an indication that the participant is on-task and therefore, appropriately attending to the stimuli.

\section{EXECUTIVE FUNCTION}

Performance indices for the Stroop test and the Go-NoGo test, and mean accuracy for the Catch Game test.

All outcome parameters were calculated automatically by using the custom software of the computerized Mindstreams battery test, and were blind to the treatment condition. 


\section{SESSIONS 2, 3, AND 4 (EXPERIMENTAL SESSIONS)}

Laboratory visits 2, 3, and 4 were counter-balanced to minimize any order or learning effects. During these visits, the participants completed the cognitive tests prior to and immediately after (within 5 minutes) the experimental intervention condition, which consisted of a 30 min aerobic exercise session, a $30 \mathrm{~min}$ resistance exercise session, or a $30 \mathrm{~min}$ rest in a seated position, in a quiet room, for the control session.

\section{AEROBIC CYCLE EXERCISE SESSION}

This session began with a 3 min warm-up of pedaling with no load. The load was then increased until the participants reached their predetermined training load of $60 \%$ of maximal load, and they continued cycling for $30 \mathrm{~min}$. This exercise session lasted approximately $40 \mathrm{~min}$ and could be described as a typical moderate-intensity aerobic exercise session, based on classifications proposed by the ACSM in 2010 (ACSM et al., 2010). The rated perceived exertion (RPE, Borg, 1974) for all participants during the aerobic session was in the range of 1214. The scale ranges from 6 (no exertion at all) to 20 (extremely hard exertion).

\section{RESISTANCE EXERCISE SESSION}

This was designed to represent a typical moderate-intensity resistance exercise training session. The session began with a warm-up of stretching exercises. Then the participants performed the resistance exercises by completing three sets of 15 repetitions at $60 \%$ of their $1 R M$, for each of the six major muscle groups that were assessed in the first visit. The exercises were performed using a Body-Solid EXM3000S multi-station gym. Participants were given a 1 min rest between each set of 15 repetitions and prior to moving on to the next muscle group. At the end of this session, a 3 min cool-down was executed by walking

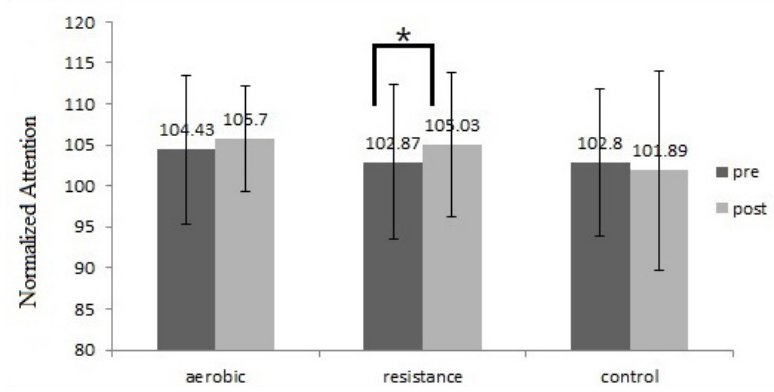

FIGURE 1.

Mean normalized scores of calculated attention before and after . slowly to the room were the cognitive tests were performed. This session lasted between 35-40 min.

\section{CONTROL SESSION}

During this condition, the participants sat quietly for $30 \mathrm{~min}$.

\section{Data Analysis}

The statistical analysis consisted of two-way analyses of variance (ANOVAs) with repeated measures: 3 conditions (aerobic exercise, resistance exercise, control) $\times 2$ times (pretest, post-test) were performed for each cognitive task. The required assumptions of normality for the parametric statistical procedure were met.

\section{RESULTS}

A significant treatment $\times$ time interaction was found for attention scores, $F(2,37.85)=3.2, p=.050$. A post-hoc test revealed a significant increase of attention scores following the resistance session (pre-post, $p$ $=.016)$. Figure 1 presents mean normalized scores of calculated attention test results before and after each intervention.

A significant treatment $\times$ time interaction was also found for $\mathrm{EF}$ scores, $F(2,35.12)=3.36, p=.046$. A post-hoc test revealed a statistically significant pre-post increase of EF scores after the resistance session, $\mathrm{p}=.026$, as well as after the aerobic session, $p=.05$. Figure 2 presents means of EF scores before and after each intervention.

\section{DISCUSSION}

The purpose of the current study was to increase the available data on the acute effects of typical moderate resistance and aerobic exercise

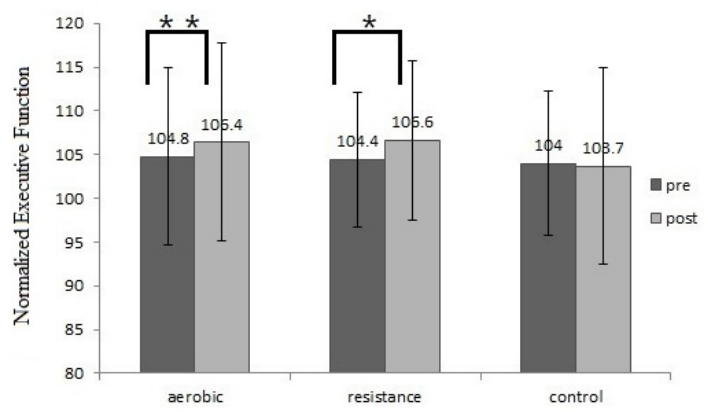

|FIGURE 2.

Means of normalized executive function scores before and after each intervention. ${ }^{*}=$ resistance session, pre-post difference, $p=.026 ;^{* *}=$ aerobic session, pre-post difference, $p=.05$.

\begin{tabular}{|c|c|c|c|c|c|c|}
\hline & \multicolumn{2}{|c|}{ Aerobic } & \multicolumn{2}{|c|}{ Resistance } & \multicolumn{2}{|c|}{ Control } \\
\hline & Pre & Post & Pre & Post & Pre & Post \\
\hline & Mean \pm SD & Mean $\pm S D$ & Mean \pm SD & Mean \pm SD & Mean $\pm S D$ & Mean \pm SD \\
\hline Attention & $104.43 \pm 9.05$ & $105.70 \pm 6.42$ & $102.87 \pm 9.44$ & $105.03 \pm 8.73$ & $102.80 \pm 8.94$ & $101.89 \pm 12.13$ \\
\hline $\begin{array}{l}\text { Executive } \\
\text { function }\end{array}$ & $104.8 \pm 10.1$ & $106.4 \pm 11.3$ & $104.4 \pm 7.7$ & $106.6 \pm 9.1$ & $104.0 \pm 8.2$ & $103.7 \pm 11.3$ \\
\hline
\end{tabular}


sessions on cognitive function of young and healthy participants accustomed to physical exercise. Our main finding was that both resistance and aerobic exercise enhanced EF and attention in young healthy adults. Attention was significantly affected only by the resistance exercise session. Our results are in line with two studies, one conducted with middle aged women (Alves et al., 2012) and the other with high school students (Harveson et al., 2016), both of which demonstrated an improvement in EF following both aerobic and resistance exercise. The fact that acute aerobic exercise improves EF is well documented (e.g., Byun et al., 2014; Tam, 2013).

It is also interesting to note that, using a similar protocol but with a mixed group of middle-aged men and women, Dunsky et al. (2017) found increased scores of attention following an aerobic exercise session on a treadmill, but not after a strength session. They also found only marginally significant improvement in executive function scores following both aerobic exercise and resistance exercise sessions. However, Pontifex et al. (2009) did not observe improvements following acute resistance exercise. This may be attributed to the greater exercise intensity applied ( $80 \%$ of $1 \mathrm{RM})$, which resulted in a deterioration of the effect.

Johnson et al. (2016) compared the cognitive performance of older adults aged 71-72 years, using the Stroop test before and after an acute aerobic or resistance exercise session, and concluded that: "independent of mode or duration of exercise, the participants improved in the Stroop Inhibition task immediately post-exercise" (p. 2).

Resistance exercises were previously shown to increase plasma cortisol (Doma et al., 2015) and noradrenalin levels (Kliszczewicz et al., 2016). The effects of the increased sympathetic system may explain the positive effects of this exercise mode, in our study as well as in others (e.g., Chang, Ku et al., 2012; Chang, Tsai, Huang, Wang, \& Chu, 2014).

Another possible mechanism that was suggested for the cognitive improvement following aerobic exercise is that exercise induces physical stress, activating both the sympathetic system and the hypothalamic pituitary-adrenal (HPA) axis (Mastorakos, Pavlatou, DiamantiKandarakis, \& Chrousos, 2005). Stress hormones related to the activation of the HPA axis and the sympathetic system, such as cortisol and noradrenalin, respectively, were shown to affect cognitive function (Lambourne \& Tomporowski, 2010; Segal, Cotman, \& Cahill, 2012).

Referring specifically to attention, it should be noted that while the effect of aerobic exercise was only marginally significant in our study, the effect of resistance exercise was found to be clearly significant. To the best of our knowledge, this is the only study showing an effect of resistance exercise on attention outcomes in young, healthy participants. It should, however, be noted that attention shares some features with EF, in that most EF tasks also require attention (Strauss, Sherman, \& Spreen, 2006). The present results indicate that indeed both acute aerobic and strength exercise sessions may positively enhance cognitive functions immediately post-exercise. However, the actual effects of an acute exercise bout on performance in life situations, such as taking a test in an academic setting or performing a demanding, novel manual task in a work setting, need further study. One of the relevant controls of such an investigation should be to practice the actual criterion task for the same duration as the acute exercise session before the testing. Also, the possible accumulative cognitive effects of repeated sessions of strength training, which result in established physiological and metabolic adaptations, need to be studied.

\section{CONCLUSION}

The present study indicates that an acute bout of a typical moderateintensity resistance training session may positively influence cognitive functions in young, healthy participants. It is possible that adding strength training sessions to a chronic aerobic training program may enhance the cognitive benefits, but this should be tested in a suitable study design. The positive effects of resistance exercise on EF and attention may provide an additional incentive for practicing this type of exercise.

\section{ACKNOWLEDGEMENTS}

We sincerely thank Ms. Dina Olswang for offering comments on earlier drafts of this paper.

\section{CONFLICTS OF INTEREST}

We declare no conflicts of interest including financial agreements or consultant relationships with organizations involved in the research.

\section{REFERENCES}

Alves, C. R., Gualano, B., Takao, P. P., Avakian, P., Fernandes, R. M., Morine, D., \& Takito, M. Y. (2012). Effects of acute physical exercise on executive functions: a comparison between aerobic and strength exercise. Journal of Sport \& Exercise Psychology, 34, 539-549. doi: 10.1123/jsep.34.4.539 الس الس

American College of Sports Medicine, Thompson, W. R., Gordon, N. F., \& Pescatello, L. S. (2010). ACSM's guidelines for exercise testing and prescription, 8th ed. Philadelphia: Lippincott Williams \& Wilkins.

Barella, A., Etnier, L., \& Chang, Y.-K. (2010). The immediate and delayed effects of an acute bout of exercise on cognitive performance of healthy older adults. Journal of Aging and Physical Activity, 18, 87-98. doi: 10.1017/CBO9781107415324.004 السلسلس

Basso, J. C., \& Suzuki, W. A. (2017). The effects of acute exercise on mood, cognition, neurophysiology, and neurochemical pathways: A review. Brain Plasticity, 2, 127-152. doi: 10.3233/ BPL-160040 س山ा

Borg, G. A. (1974). Perceived exertion. Exercise and Sport Sciences Reviews, 2, 131-153. Retrieved from http://www.ncbi.nlm.nih. gov/pubmed/4466663

Bullock, T., \& Giesbrecht, B. (2014). Acute exercise and aerobic fitness influence selective attention during visual search. Frontiers in Psychology, 5, 1290. doi: 10.3389/fpsyg.2014.01290 السلسلسلس

Byun, K., Hyodo, K., Suwabe, K., Ochi, G., Sakairi, Y., Kato, M., ... Soya, H. (2014). Positive effect of acute mild exercise on executive function via arousal-related prefrontal activations: An 
fNIRS study. Neurolmage, 98, 336-345. doi: 10.1016/j.neuroimage.2014.04.067 السلسلس

Chang, Y.-K., Ku, P.-W., Tomporowski, P. D., Chen, F.-T., \& Huang, C.-C. (2012). Effects of acute resistance exercise on late-middle-age adults' goal planning. Medicine and Science in Sports and Exercise, 44, 1773-1779. doi: 10.1249/MSS.0b013e3182574e0b الإلسلس الس الس

Chang, Y.-K., Pesce, C., Chiang, Y.-T., Kuo, C.-Y., \& Fong, D.-Y. (2015). Antecedent acute cycling exercise affects attention control: An ERP study using attention network test. Frontiers in Human Neuroscience, 9, 156.doi: 10.3389/fnhum.2015.00156 سلس

Chang, Y.-K., Tsai, C.-L., Huang, C.-C., Wang, C.-C., \& Chu, I.-H. (2014). Effects of acute resistance exercise on cognition in late middle-aged adults: General or specific cognitive improvement? Journal of Science and Medicine in Sport, 17, 51-55. hdoi

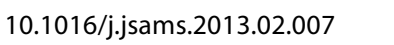

Chang, Y., \& Etnier, J. (2009). Effects of an acute bout of localized resistance exercise on cognitive performance in middle-aged adults: A randomized controlled trial study. Psychology of Sport and Exercise, 10, 19-24. doi: 10.1016/j.psychsport.2008.05.004

Chang, Y. K., Labban, J. D., Gapin, J. I., \& Etnier, J. L. (2012). The effects of acute exercise on cognitive performance: A metaanalysis. Brain Research, 1453, 87-101. doi: 10.1016/j.brainres.2012.02.068 الم

Doma, K., Schumann, M., Sinclair, W. H., Leicht, A. S., Deakin, G. B., \& Häkkinen, K. (2015). The repeated bout effect of typical lower body strength training sessions on sub-maximal running performance and hormonal response. European Journal of Applied Physiology, 115, 1789-1799. doi: 10.1007/s00421015-3159-z

Doniger, G. M., Simon, E. S., \& Schweiger, A. (2008). Adjustment of cognitive scores with a co-normed estimate of premorbid intelligence: implementation using mindstreams computerized testing. Applied Neuropsychology, 15, 250-263. doi: 10.1080/09084280802325074 الس الس الس

Dunsky, A., Abu-Rukun, M., Tsuk, S., Dwolatzky, T., Carasso, R., \& Netz, Y. (2017). The effects of a resistance vs. an aerobic single session on attention and executive functioning in adults. PLOS One, 12, e0176092. doi: 10.1371/journal.pone.0176092 السلالسلس

Dwolatzky, T., Whitehead, V., Doniger, G. M., Simon, E. S., Schweiger, A., Jaffe, D., \& Chertkow, H. (2003). Validity of a novel computerized cognitive battery for mild cognitive im-

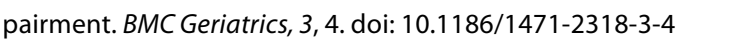

Fiatarone Singh, M. A., Gates, N., Saigal, N., Wilson, G. C., Meiklejohn, J., Brodaty, H., ... Valenzuela, M. (2014). The Study of Mental and Resistance Training (SMART) Study-Resistance training and/or cognitive training in mild cognitive impairment: A randomized, double-blind, double-sham controlled trial. Journal of the American Medical Directors Association, 15,

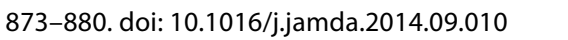

Fleck, S. J., \& Kraemer, W. J. (2014). Designing resistance training programs (4th ed.). Champaign, IL: Human Kinetics.

Halperin, J., Wolf, L.,Greenblatt, E. R., \& Young, G. (1991). Subtype analysis of commission errors on the continuous performance test in children. Developmental Neuropsychology, 7, 207-217.

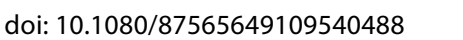

Harveson, A. T., Hannon, J. C., Brusseau, T. A., Podlog, L., Papadopoulos, C., Durrant, L. H., ... Kang, K.-D. (2016). Acute effects of 30 minutes resistance and aerobic exercise on cognition in a high school sample. Research Quarterly for Exercise and Sport, 87, 214-220. doi: 10.1080/02701367.2016.1146943 الس السلس Hogervorst, E., Riedel, W., Jeukendrup, A., \& Jolles, J. (1996). Cognitive performance after strenuous physical exercise. Perceptual and Motor Skills, 83, 479-488. doi: 10.2466/ pms.1996.83.2.479 السلسل

Johnson, L., Addamo, P. K., Selva Raj, I., Borkoles, E., Wyckelsma, V., Cyarto, E., \& Polman, R. C. (2016). An acute bout of exercise improves the cognitive performance of older adults. Journal of Aging and Physical Activity, 24, 591-598. doi: 10.1123/

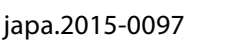

Kliszczewicz, B. M., Esco, M. R., Quindry, J. C., Blessing, D. L., Oliver, G. D., Taylor, K. J., \& Price, B. M. (2016). Autonomic responses to an acute bout of high-intensity body weight resistance exercise vs. treadmill running. The Journal of Strength \& Conditioning Research, 30, 1050-1058. doi: 10.1519/JSC.0000000000001173 السلسلس

Knuttgen, H. G. (2007). Strength training and aerobic exercise: Comparison and contrast. The Journal of Strength and Conditioning Research, 21, 973-978. doi: 10.1519/R-505011.1 لسلسلس

Lambourne, K., \& Tomporowski, P. (2010). The effect of exerciseinduced arousal on cognitive task performance: A metaregression analysis. Brain Research, 1341, 12-24. doi: 10.1016/j. brainres.2010.03.091 1لس

Mastorakos, G., Pavlatou, M., Diamanti-Kandarakis, E., \& Chrousos, G. P. (2005). Exercise and the stress system. Hormones, 4, 73-89. Retrieved from http://www.ncbi.nlm.nih.gov/pubmed/16613809

Netz, Y., Abu-Rukun, M., Tsuk, S., Dwolatzky, T., Carasso, R., Levin, O., \& Dunsky, A. (2016). Acute aerobic activity enhances response inhibition for less than $30 \mathrm{~min}$. Brain and Cognition, 109, 59-65. doi: 10.1016/j.bandc.2016.08.002 1ل

Pontifex, M. B., Hillman, C. H., Fernhall, B., Thompson, K. M., \& Valentini, T. A. (2009). The effect of acute aerobic and resistance exercise on working memory. Medicine and Science in Sports and Exercise, 41, 927-934. doi: 10.1249/ MSS.0b013e3181907d69 الس الس الس الس

Segal, S. K., Cotman, C. W., \& Cahill, L. F. (2012). Exercise-induced noradrenergic activation enhances memory consolidation in both normal aging and patients with amnestic mild cognitive impairment. Journal of Alzheimer's Disease, 32, 1011-1018. doi: 10.3233/JAD-2012-121078 سلس لس

Strauss, E., Sherman, E. M. S., \& Spreen, O. (2006). A compendium of neuropsychological tests: Administration, norms, and commentary. Oxford, UK: Oxford University Press. Retrieved from https://books.google.com/books?id=jQ7n4QVw7-0C\&pgis=1

Tam, N. D. (2013). Improvement of processing speed in executive 
function immediately following an increase in cardiovascular activity. Cardiovascular Psychiatry and Neurology, 212767. doi: 10.1155/2013/212767 الس الس الس

Tine, M. (2014). Acute aerobic exercise: An intervention for the selective visual attention and reading comprehension of low-income adolescents. Frontiers in Psychology, 5, 575. doi: 10.3389/fpsyg.2014.00575 السلسلس
Zimmer, P., Stritt, C., Bloch, W., Schmidt, F.-P., Hübner, S. T., Binnebößel, S., ... Oberste, M. (2016). The effects of different aerobic exercise intensities on serum serotonin concentrations and their association with Stroop task performance: a randomized controlled trial. European Journal of Applied Physiology, 116, 2025-2034. doi: 10.1007/s00421-016-3456-1 10ل

RECEIVED 13.07.2017 | ACCEPTED 24.07.2019 


\section{APPENDIX}

\begin{tabular}{|c|c|c|c|c|c|c|c|c|c|c|c|c|}
\hline & \multicolumn{4}{|c|}{ Aerobic } & \multicolumn{4}{|c|}{ Resistance } & \multicolumn{4}{|c|}{ Control } \\
\hline & \multicolumn{2}{|c|}{ Pre } & \multicolumn{2}{|c|}{ Post } & \multicolumn{2}{|c|}{ Pre } & \multicolumn{2}{|c|}{ Post } & \multicolumn{2}{|c|}{ Pre } & \multicolumn{2}{|c|}{ Post } \\
\hline & $\mathbf{M}$ & SD & $\mathbf{M}$ & SD & $\mathbf{M}$ & SD & $\mathbf{M}$ & SD & $\mathbf{M}$ & SD & $\mathbf{M}$ & SD \\
\hline Attention & 104.43 & 9.05 & 105.70 & 6.42 & 102.87 & 9.44 & 105.03 & 8.73 & 102.80 & 8.94 & 101.89 & 12.13 \\
\hline Executive function & 104.8 & 10.1 & 106.4 & 11.3 & 104.4 & 7.7 & 106.6 & 9.1 & 104.0 & 8.2 & 103.7 & 11.3 \\
\hline Go-NoGo reaction time & 102.74 & 10.55 & 104.76 & 8.23 & 101.61 & 9.91 & 103.65 & 8.85 & 102.15 & 10.58 & 100.31 & 13.75 \\
\hline Go-NoGo composite score & 104.09 & 13.59 & 105.95 & 16.31 & 102.71 & 11.48 & 106.10 & 12.39 & 102.95 & 11.80 & 100.37 & 17.97 \\
\hline Go-NoGo omission errors & 101.27 & 5.96 & 100.66 & 12.66 & 102.62 & 3.11 & 102.52 & 3.78 & 101.63 & 5.24 & 100.62 & 12.76 \\
\hline Go-NoGo commission errors & 103.81 & 13.68 & 104.05 & 15.62 & 104.71 & 12.15 & 106.21 & 10.76 & 103.38 & 11.85 & 102.87 & 17.50 \\
\hline Go-NoGo commission response time & 100.04 & 11.76 & 103.33 & 6.85 & 102.65 & 7.36 & 104.40 & 4.62 & 99.65 & 7.74 & 100.90 & 7.59 \\
\hline Stroop level 1 accuracy & 104.34 & .45 & 100.96 & 12.32 & 103.05 & 8.24 & 99.67 & 14.51 & 93.12 & 23.04 & 100.47 & 13.89 \\
\hline Stroop level 1 , response time & 111.55 & 8.61 & 113.67 & 7.28 & 110.78 & 11.07 & 112.80 & 8.65 & 111.64 & 9.74 & 111.90 & 9.82 \\
\hline Stroop level 1, SD & 106.38 & 3.65 & 106.12 & 4.60 & 105.43 & 8.38 & 106.68 & 4.42 & 106.95 & 3.99 & 106.03 & 6.80 \\
\hline Stroop level 1, composite score & 115.84 & 14.39 & 118.40 & 13.67 & 115.14 & 15.79 & 116.81 & 13.15 & 114.04 & 15.42 & 116.12 & 15.76 \\
\hline Stroop level 2, accuracy & 96.30 & 20.31 & 101.52 & 11.50 & 97.61 & 16.88 & 100.63 & 13.97 & 102.55 & 10.11 & 101.10 & 11.41 \\
\hline Stroop level 2, response time & 109.04 & 11.01 & 108.45 & 11.26 & 106.97 & 14.12 & 109.55 & 11.52 & 106.22 & 13.32 & 107.02 & 13.56 \\
\hline Stroop level 2, SD & 104.43 & 10.09 & 103.50 & 11.28 & 103.70 & 12.63 & 103.70 & 13.15 & 101.05 & 16.18 & 102.45 & 13.11 \\
\hline Stroop level 2, composite score & 109.95 & 15.47 & 110.24 & 13.88 & 108.04 & 15.77 & 112.01 & 15.96 & 107.94 & 14.78 & 108.77 & 14.26 \\
\hline Stroop level 3, accuracy & 100.30 & 13.90 & 101.34 & 12.33 & 100.74 & 7.45 & 102.74 & 4.25 & 101.87 & 5.11 & 101.75 & 10.50 \\
\hline Stroop level 3, response time & 103.89 & 12.97 & 105.30 & 13.85 & 105.35 & 4.83 & 106.61 & 5.46 & 104.59 & 7.03 & 103.40 & 13.98 \\
\hline Stroop level 3, SD & 103.25 & 9.02 & 103.33 & 13.22 & 104.58 & 4.03 & 104.59 & 4.25 & 102.83 & 8.90 & 102.01 & 14.04 \\
\hline Stroop level 3, composite score & 106.17 & 16.85 & 110.71 & 14.03 & 106.70 & 11.09 & 111.04 & 10.62 & 107.04 & 10.67 & 107.94 & 14.53 \\
\hline Catch game time to make first move & 105.04 & 11.24 & 106.28 & 16.81 & 103.86 & 15.84 & 107.28 & 10.25 & 104.33 & 10.99 & 106.50 & 11.40 \\
\hline Catch game first move response time SD & 100.95 & 11.57 & 102.59 & 16.79 & 101.00 & 15.25 & 101.53 & 13.10 & 100.66 & 10.46 & 101.47 & 12.64 \\
\hline $\begin{array}{l}\text { Catch game average number of direction } \\
\text { changes per trial }\end{array}$ & 95.28 & 14.92 & 99.81 & 13.08 & 99.24 & 15.95 & 97.05 & 18.20 & 100.31 & 15.03 & 101.13 & 16.51 \\
\hline Catch game total score & 104.20 & 10.30 & 102.40 & 14.11 & 103.92 & 11.07 & 102.70 & 12.76 & 101.93 & 11.39 & 102.93 & 10.49 \\
\hline Catch game average error on missed trials & 103.24 & 8.72 & 103.21 & 11.21 & 103.50 & 9.46 & 102.36 & 11.28 & 102.77 & 9.19 & 102.91 & 8.94 \\
\hline
\end{tabular}

\section{MEASURED COGNITIVE PARAMETERS}

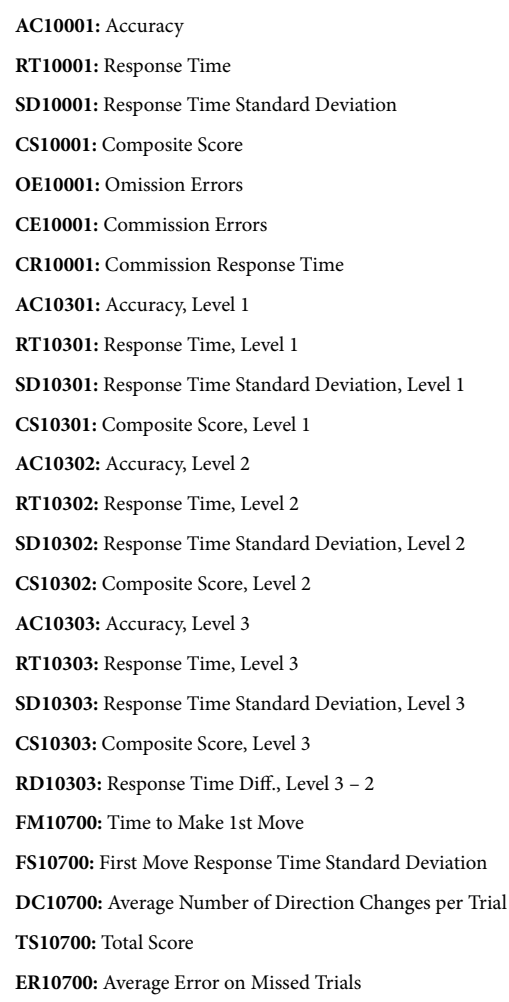

